\title{
Folk Physics for Crows?
}

\author{
Alex H. Taylor \\ School of Psychology, The University of Auckland, New Zealand \\ *Corresponding author (Email: alexander.taylor@auckland.ac.nz) \\ Citation - Taylor, A.H. (2020). Folks physics for crows? Animal Behavior and Cognition, 7(3), 452-456. doi: \\ https://doi.org/10.26451/abc.07.03.11.2020

\begin{abstract}
New Caledonian crows are a key model species for testing whether the ability to reason about unobservable causal mechanisms is unique to humans. Here, I review the growing body of evidence for the absence of this ability in this species.
\end{abstract}

Keywords - New Caledonian crows, Folk physics, Causal understanding, Causal reasoning, Convergent evolution

It is remarkable to think that at the time of publication of Folk Physics for Apes (Povinelli, 2000), there were no studies on the causal understanding of birds. Avian cognition at the time was just beginning to emerge as a discipline (Clayton \& Dickinson, 1998; Pepperberg et al., 1997), as was the news that chimpanzees might not be the most proficient animal tool-maker outside of humans, due to the behavior of the New Caledonian (NC) crow (Hunt et al., 1996, 2000).

How times have changed. Today, avian cognition is a well-established field (e.g., Auersperg \& Von Bayern, 2019; Güntürkün \& Bugnyar, 2016; Taylor, 2014) and the NC crow has been the focus of many studies aiming to probe this bird's understanding of causality, due to its ability to use and manufacture tools from a range of materials for extractive foraging and other purposes (Hunt, 1996; StClair et al., 2018; Taylor, Hunt, et al., 2012; von Bayern et al., 2018; Wimpenny et al., 2011). Research on NC crow cognition offers a chance to test the ubiquity of the central thesis of Folk Physics for Apes, that

...chimpanzees detect the regularities that exist between events, and learn to act on the basis of them, but they do not appeal to unobservable phenomena (force, gravity, etc.) to account for (or assist in their reasoning about) such regular associations of events. (Povinelli 2000, p. 298)

NC crows are an excellent model species for testing this hypothesis, given the suggested link between complex tool behaviors and the evolution of intelligence (the technical intelligence hypothesis, Byrne, 1997). If there is one species that we might expect to have evolved more complex technical intelligence than our closest relatives, it would be the one that shows more sophisticated types of wild tool behaviors than them, such as hook crafting (Taylor \& Gray, 2014). Thus, research on the cognition of $\mathrm{NC}$ crows offers one route towards testing whether Povinelli's hypothesis holds not only for chimpanzees but, more generally, for other animals as well.

The key question, then, is whether NC crows show the same limitation as chimpanzees, in that they attend to the regularities of the world, but do not infer the presence of unobservable causal forces. Research focused on answering this question initially presented NC crows with the trap-tube problem, where an animal needs to pull food out of a horizontal tube while avoiding a trap set into the surface of the tube (Taylor, Hunt et al., 2009; Taylor, Roberts et al., 2009). Of the six NC crows tested, three learned 
to solve an initial trap-tube problem and then solved several transfer tasks, where obvious, but irrelevant features of the initial tube, such as the rim of the hole being blue, were removed. NC crows then successfully passed the inverted tube control, where the initial trap-tube used was turned upside down, making the trap non-functional, as objects cannot fall up. Crows then solved the trap-table task, a problem with an identical causal structure to the trap-tube task (in that successful performance requires avoiding pulling food into a hole), but with very different perceptual elements.

The performance of these birds was highly impressive as apes often struggle to transfer performance from the trap-tube to the trap-table while using tools (Martin-Ordas et al., 2008). These results show that NC crows can attend to causal regularities and go beyond the law of stimulus generalization (Shepard, 1987), by forming abstract categories not tied to specific perceptual features. The key question, however, is whether this abstract knowledge was based on the observable features of the problem, such as surface continuity, or of the unobservable causal mechanism that made these features relevant, namely gravity (Vonk \& Povinelli, 2006). Importantly, the NC crows failed to solve a trap-tube transfer task that contained two holes, only one of which had a base and so worked as a functional trap. The presence of this error demonstrates that these crows were unable to switch from avoiding the hole in a trap-tube, to avoiding the base of a trap when two holes were present. This suggests, as Taylor, Hunt, et al. (2009) noted, that

...the crows had knowledge of the meat's relation with the hole as a proximate causal regularity but did not infer the existence of a distal causal mechanism (gravity). If the crows were capable of more sophisticated causal reasoning, they would have viewed the meat's relations with the hole and the trap base as two separate proximate causal regularities that were variants of the same distal causal mechanism (gravity). This would have led to a spontaneous sensitivity to the trap base as a causal feature. (p. 6)

Thus, these results offer support for Povinelli's hypothesis: NC crows appeared to have causal understanding of the observable features of the trap-tube problem, but not the unobservable mechanism, namely gravity.

A second line of research on this species has also found a similar pattern of results. Von Bayern et al. (2009) demonstrated that, after limited experience pushing a platform with their beak, NC crows will, when the platform is moved out-of-reach, spontaneously drop stones onto the platform to trigger it. This impressive problem solving can be accounted for by two hypotheses (Seed \& Byrne, 2010; Shettleworth, 2010; von Bayern et al., 2009), one explaining this behavior as the product of an understanding of force, the other an understanding of contact. In the force account, the birds learn from pushing the platform with their beak that a sufficient amount of force needs to be applied to the platform to trigger it and then attempt to recreate this causal force by dropping objects external to their body down the tube. In the contact account, the birds learn that contact between their beak and the platform leads to it triggering and then attempt to recreate this causal relation by dropping objects external to their body down the tube. This latter hypothesis predicts animals should make a crucial mistake, the weight inattention error, when dropping objects. If the birds are attempting only to make contact with the platform, rather than hitting it with sufficient force to trigger it, they should be insensitive to the weight of the object they are dropping.

Anecdotal support for this error came from observations by von Bayern et al. (2009) of instances where the NC crows attempted to drop a feather down the tube. Neilands et al. (2016) then found clear evidence of this error by presenting another group of NC crows with a different variant of the task. As in the von Bayern study, crows were initially given experience of triggering the platform with their beak. At test, the platform was moved out-of-reach and crows were given objects of two different colors to drop down the tube, with objects of one color being heavy, and so capable of triggering the platform, while objects of the other color were very light, and so incapable of triggering the platform. If the NC crows understood that force needed to be applied to the platform, they should have shown a preference for the heavy objects. However, no preference was shown by any of the crows solving the task, which suggests 
that the solutions seen were due to crows attempting to make contact with the platform, rather than attempting to exert sufficient force on the platform to make it trigger.

So, does Povinelli's hypothesis extend to New Caledonian crows? Evidence from these two lines of research enquiry suggests these crows can reason causally about observable features of the world, such as moving objects along surfaces and making contact with platforms, but do not make deeper inferences abut unobservable causal mechanisms such as gravity and force. In each of these paradigms, NC crows used their experience of how apparatuses worked in a highly flexible way to solve novel problems, transferring from the trap-tube to the trap table (Taylor, Hunt et al., 2009; Taylor, Roberts et al., 2009), and transferring from pushing a platform with their beak to dropping stones onto a platform (Von Bayern et al., 2009). However, when these performances were probed further, no evidence emerged of the crows understanding why these apparatuses worked as they did, in terms of the unobservable causal mechanisms at play. This is in line with Povinelli's observations of chimpanzee problem solving.

However, it seems hasty to conclude from these observations alone that NC crows are entirely incapable of reasoning about unobservable causal mechanisms. First, it is unclear whether researchers have created tasks that can directly answer Povinelli's hypothesis. Consider Taylor, Miller, et al. (2012), a study that tested if NC crows could infer that the movement of a stick from a hide was caused by a human they had seen enter the hide. The crows seemed able to 'join the dots' and infer that a hidden causal mechanism (the hidden human), was the cause of the stick's movement. Though associative accounts for the crows' performance have been proposed (Boogert et al., 2013; Dymond et al., 2013) they do not seem able to explain the results in their entirety (Taylor et al., 2013a, b). Irrespective of this debate, this study raises a key point concerning the design of many of the tests used to examine Povinelli's hypothesis. Many paradigms so far created have tested if animals can use observable causal cues to solve a problem, and then examined if animals are also making inferences about unobservable causal mechanisms with the same problem. There is, therefore, a large jump in the complexity of the problems being presented. Perhaps a fairer strategy would be to use different versions of the same paradigm to test if an animal can 1) make any kind of inference about a hidden object, for example during object permanence tests, where there is a well-developed literature (see Jaakkola, 2014, for a review), 2) make an inference about a hidden causal mechanism (e.g., a human who disappears from view and then creates an observable effect) and then 3) makes an inference about an unobservable causal mechanism (e.g., an observable effect caused by gravity in the same context). Such a ratcheting up in complexity of the inferences required across a series of highly similar situations would provide much more convincing evidence that unanticipated variables are not hindering performance, due to the inclusion of the baseline tests of reasoning about hidden objects and agents/mechanisms. This would directly test if it is the lack of an ability to make an inference about unobservable causal mechanisms, rather than the more general lack of an ability to make inference about any hidden mechanism, that is a key difference between human and animal minds.

A more general concern is that animals can produce very different performances when faced with different types of experiment attempting to test for the same cognitive mechanism. Early work on the trap-tube problem showed that animal performance improved considerably if animals could pull food towards them with a tool, rather than push it away (Mulcahy \& Call, 2006). Similarly, while Povinelli has suggested, based on his experiments on weight with chimpanzees, that only humans have a causal understanding of weight (Povinelli, 2012), recent work on NC crows, using a novel paradigm, has shown that they have the ability to use their observations of objects moving in the wind to determine their weight (Jelbert et al., 2019). Just within NC crows, tests of mental simulation, another key aspect of causal cognition, suggested this species could not mentally simulate a man-made object (string) (Taylor et al., 2010, Taylor, Knaebe et al., 2012), yet recent work has provided clear evidence that this species can preplan solutions to other tool problems via mental simulation (Gruber et al., 2019).

In each of these instances, it would have been possible to use the available evidence to underestimate the cognitive abilities of animals, based on the limitations researchers imposed on the animals being tested, in terms of either the behavior they could use (Mulcahy \& Call, 2006), the materials they could interact with (Taylor et al., 2010; Taylor, Knaebe et al., 2012), or the structure of the task 
(Jelbert et al., 2019). Thus, while there is a growing body of evidence for the absence of the ability to reason about unobservable causal mechanisms in $\mathrm{NC}$ crows, there is also mounting evidence showing animal performance can vary dramatically based on the type of problem presented. It, therefore, seems prudent to be cautious in interpreting the body of evidence generated to date. Clearly, at present, there does not seem to be any evidence that $\mathrm{NC}$ crows reason about unobservable causal mechanisms. However, it seems too early to interpret this pattern of results as evidence that such reasoning is beyond the capacity of the NC crow mind entirely. The search for animal folk physics requires we avoid our own bias for jumping to conclusions too hastily.

\section{References}

Auersperg, A. M. I., \& von Bayern, A. M. P. (2019). Who's a clever bird - now? A brief history of parrot cognition. Behaviour, 156, 391-407.

Boogert, N. J., Arbilly, M., Muth, F., \& Seed, A. M. (2013). Do crows reason about causes or agents? The devil is in the controls. Proceedings of the National Academy of Sciences, 110, E273-E273.

Byrne, R. W. (1997). The technical intelligence hypothesis: an additional evolutionary stimulus to intelligence? In A. Whiten \& R. Byrne (Eds.), Machiavellian intelligence II (pp. 289-311). Cambridge University Press.

Clayton, N. S., \& Dickinson, A. (1998). Episodic-like memory during cache recovery by scrub jays. Nature, 395, 272-274.

Dymond, S., Haselgrove, M., \& McGregor, A. (2013). Clever crows or unbalanced birds? Proceedings of the National Academy of Sciences of the United States of America, 110, E336.

Gruber, R., Schiestl, M., Boeckle, M., Frohnwieser, A., Miller, R., Gray, R. D., Clayton, N. S., \& Taylor, A. H. (2019). New Caledonian crows use mental representations to solve metatool problems. Current Biology, $29,686-692$.

Güntürkün, O., \& Bugnyar, T. (2016). Cognition without cortex. Trends in Cognitive Science, 20, 291-303.

Hunt, G. R. (1996). Manufacture and use of hook-tools by New Caledonian crows. Nature, 379, 249-251.

Hunt, G. R. (2000). Human-like, population-level specialization in the manufacture of pandanus tools by New Caledonian crows (Corvus moneduloides). Proceedings of the Royal Society of London. Series B: Biological Sciences, 267, 403-413.

Jaakkola, K. (2014). Do animals understand invisible displacement? A critical review. Journal of Comparative Psychology, 128, 225-239.

Jelbert, S. A., Miller, R., Schiestl, M., Boeckle, M. Cheke, L.G., Gray, R.D., Taylor, A.H., \& Clayton, N. S. (2019) New Caledonian crows infer the weight of objects from observing their movements in a breeze. Proceedings of the Royal Society of London Series B: Biological Sciences, 286, 20182332.

Martin-Ordas, G., Call, J., \& Colmenares, F. (2008). Tubes, tables and traps: Great apes solve two functionally equivalent trap tasks but show no evidence of transfer across tasks. Animal Cognition, 11, 423-430.

Mulcahy, N. J., \& Call, J. (2006). How great apes perform on a modified trap-tube task. Animal Cognition, 9, 193199.

Neilands, P. D., Jelbert, S. A., Breen, A. J., Schiestl, M., \& Taylor, A. H. (2016). How insightful is 'insight'? New Caledonian crows do not attend to object weight during spontaneous stone dropping. PloS One, 11, e0167419.

Pepperberg, I. M., Willner, M. R., \& Gravitz, L. B. (1997). Development of Piagetian object permanence in grey parrot (Psittacus erithacus). Journal of Comparative Psychology, 111, 63-75.

Povinelli, D. (2012). World without weight: Perspectives on an alien mind. Oxford University Press.

Povinelli, D. (2000). Folk physics for apes: The chimpanzee's theory of how the world works. Oxford University Press.

Seed, A., \& Byrne, R. (2010). Animal tool-use. Current Biology, 20, R1032-R1039.

Shettleworth, S. J. (2010). Clever animals and killjoy explanations in comparative psychology. Trends in Cognitive Science, 14, 477-481.

Shepard, R. N. (1987). Toward a universal law of generalization for psychological science. Science, 237, 13171323.

Taylor, A. H. (2014). Corvid cognition. Wiley Interdisciplinary Reviews: Cognitive Science, 5, 361-372.

Taylor, A. H., Cheke, L. G., Waismeyer, A., Meltzoff, A. N., Miller, R., Gopnik, A., Clayton, N. S. \& Gray, R. D. (2014). Of babies and birds: Complex tool behaviours are not sufficient for the evolution of the ability to create a novel causal intervention. Proceedings of the Royal Society: Biological Sciences, 281, 20140837. 
Taylor, A. H., Cheke, L. G., Waismeyer, A., Meltzoff, A., Miller, R., Gopnik, A., Clayton, N. S. \& Gray, R. D. (2015). No conclusive evidence that corvids can create novel causal interventions. Proceedings of the Royal Society B: Biological Sciences, 282, 20150796.

Taylor, A. H., \& Gray, R. D. (2014). Is there a link between the crafting of tools and the evolution of cognition? Wiley Interdisciplinary Reviews: Cognitive Science, 5, 693-703.

Taylor, A. H., Hunt, G. R., \& Gray, R. D. (2012). Context-dependent tool use in New Caledonian crows. Biology Letters, 8, 205-207.

Taylor, A., Hunt, G., Medina, F., \& Gray, R. (2009). Do New Caledonian crows solve physical problems through causal reasoning? Proceedings of the Royal Society B: Biological Sciences, 276, 247-254.

Taylor, A. H., Knaebe, B., \& Gray, R. D. (2012). An end to insight? New Caledonian crows can spontaneously solve problems without planning their actions. Proceedings of the Royal Society B: Biological Sciences, 279, 4977-4981.

Taylor, A. H., Medina, F. S., Holzhaider, J. C., Hearne, L. J., Hunt, G. R., \& Gray, R. D. (2010). An investigation into the cognition behind spontaneous string pulling in New Caledonian crows. PloS One, 5, e9345.

Taylor, A. H., Miller, R., \& Gray, R. D. (2012). New Caledonian crows reason about hidden causal agents. Proceedings of the National Academy of Sciences, 109, 16389-16391.

Taylor, A. H., Miller, R., \& Gray, R. D. (2013a). Reply to Boogert et al.: The Devil is unlikely to be in association or distraction. Proceedings of the National Academy of Sciences, 110, E274.

Taylor, A. H., Miller, R., \& Gray, R. D. (2013b). Reply to Dymond et al.: Clear evidence of habituation counters counterbalancing. Proceedings of the National Academy of Sciences, 110, E337.

Taylor, A., Roberts, R., Hunt, G., \& Gray, R. (2009). Causal reasoning in New Caledonian crows: Ruling out spatial analogies and sampling error. Communicative and Integrative Biology, 2, 311-312.

von Bayern, A. M. P., Danel, S., Auersperg, A. M. I., Mioduszewska, B., \& Kacelnik, A. (2018). Compound tool construction by New Caledonian crows. Scientific Reports, $8,1-8$.

von Bayern, A. M. P., Heathcote, R. J. P., Rutz, C., \& Kacelnik, A. (2009). The role of experience in problem solving and innovative tool use in crows. Current Biology, 19, 1965-1968.

Vonk, J., \& Povinelli, D. J. (2006). Similarity and difference in the conceptual systems of primates: The unobservability hypothesis. In T. Zentall \& E.A. Wasserman (Eds.), Comparative cognition: Experimental explorations of animal intelligence (pp. 363-87). Oxford University Press.

Wimpenny, J. H., Weir, A. A., \& Kacelnik, A. (2011). New Caledonian crows use tools for non-foraging activities. Animal Cognition, 14, 459-464. 